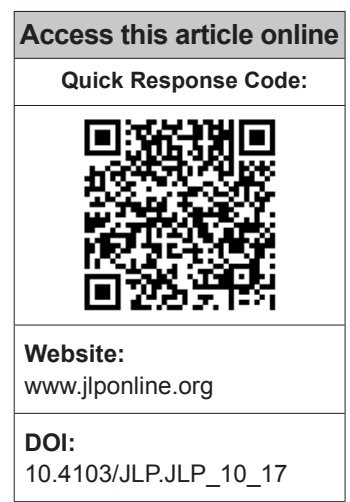

Departments of Transfusion Medicine and ${ }^{1}$ Community Medicine, Gian Sagar Medical College and Hospital, Patiala, Punjab, India

Address for correspondence: Dr. Sonam Kansay, H. No. 120 , Sector 20 A, Chandigarh - 160020 , India.

E-mail: sonu4068@ yahoo.co.in

Submission: 16-01-2017 Accepted: 26-04-2017

\title{
Effect of introduction of single-donor apheresis platelets in dengue management: A comparative analysis of two consecutive dengue epidemics
}

\author{
Sonam Kansay, Harinder Singh ${ }^{1}$
}

\section{Abstract:}

BACKGROUND: The frequency of dengue outbreak is increasing in Southeast Asian countries these days and since there are no specific drugs against the dengue virus; therefore, treatment consists of mainly symptomatic and supportive care.

AIMS: Platelet transfusion forms one of the major parts of treatment therapy in dengue; therefore, this study was initiated to elicit the effect of introduction of single-donor apheresis platelets (SDAPs) in dengue management.

SETTINGS AND DESIGN: This is a retrospective study conducted on 622 clinically suspected cases of dengue infection who received platelet transfusions between August 2013 and December 2015 in a teaching hospital of North India.

MATERIALS AND METHODS: Clinical data, reports of hematological investigation, transfusion request forms, platelet requirements, and data obtained from daily follow-up and blood bank records were analyzed using IBM SPSS version 20.

RESULTS: Average platelet count at which platelet transfusion initiated was 25,703/cumm. Average number of random donor platelets (RDPs) transfused significantly decreased in 2015 (2013 vs. 2015 is 5.4 vs. 4.3 ) due to the transfusion of SDAP to patients with very low platelet count and those with superimposed high-risk factors. Mean length of stay in hospital was similar for patients receiving RDP only and for those receiving RDP and SDAP both (5.48 vs. 5.54) while that for patients receiving SDAP only was quite lower (3.6). Overall cost of stay was higher for those receiving SDAP transfusions.

CONCLUSION: Decision for initiating platelet transfusions and calculating its dose for dengue patients is highly variable, but transfusing high-dose platelets such as SDAP at an appropriate stage can reduce further requirement of platelet transfusions, fasten the recovery, reduce the hospital stay, lower the risk of transfusion-associated adverse reactions, and can further minimize the associated morbidity and mortality.

Key words:

Dengue fever/dengue hemorrhagic fever/dengue shock syndrome, random donor platelets, single-donor apheresis platelets

\section{Introduction}

$D_{p}^{e}$ engue virus is a single-stranded positive polarity ribonucleic acid virus of Flaviviridae family and it is one of the most common causes of arboviral

This is an open access article distributed under the terms of the Creative Commons Attribution-NonCommercial-ShareAlike 3.0 License, which allows others to remix, tweak, and build upon the work non-commercially, as long as the author is credited and the new creations are licensed under the identical terms.

For reprints contact: reprints@medknow.com disease throughout the world. Dengue fever (DF)/dengue hemorrhagic fever (DHF)/dengue shock syndrome (DSS) is caused by one of the four closely related but antigenically distinct virus serotypes (DEN1, DEN2, DEN3, and DEN4), and therefore, infection with one of these

How to cite this article: Kansay S, Singh $\mathrm{H}$. Effect of introduction of single-donor apheresis platelets in dengue management: A comparative analysis of two consecutive dengue epidemics. J Lab Physicians 2018;10:173-8. 
serotypes does not provide cross-immunity; hence, persons living in a dengue-endemic area can have four different dengue infections during their lifetime. ${ }^{[1]}$ These days, the frequency of dengue outbreak is increasing in Southeast Asian Region and its contributory factors are population growth, urbanization, inadequate water management, and lack of effective mosquito control. Convenient global travel and changes in the weather patterns have helped in expansion of the habitat range of the mosquito vector. ${ }^{[2]}$

DF is characterized by the sudden onset of acute febrile illness consisting of high-grade fever, severe headache (especially in the retro-orbital area), arthralgia, myalgia, nausea, vomiting, and rash lasting approximately 2-7 days. Infants and younger children tend to present with an undifferentiated febrile disease, often with rash. The primary pathophysiology seen in DHF is an acute increase in vascular permeability leading to leakage of plasma into the extravascular compartment, resulting in hemoconcentration and decreased blood pressure. Decline in platelet count tends to precede plasma leakage, and therefore, platelet counts and packed cell volume are often used to monitor for impending deterioration. ${ }^{[3]}$ Severe plasma leakage results in prolonged shock, accompanied by metabolic acidosis, which in turn precipitates disseminated intravenous coagulation. There are no specific drugs against the dengue virus, and therefore, treatment is mainly symptomatic and supportive. Proper management of fluid balance is the basic requirement. DSS is a medical emergency associated with a very high mortality. Massive hemorrhages in DHF/DSS usually require intensive blood components transfusion support, but early identification of the plasma leakage phase with prompt resuscitation reduces complications and improves outcome. ${ }^{[2]}$

The bleeding manifestations in dengue are caused by vasculopathy, thrombocytopenia, platelet dysfunction, and coagulopathy. ${ }^{[4,5]}$ The decision to transfuse platelets is based on several factors including estimation of platelet count and function, cause of thrombocytopenia, the status of coagulation system, the presence or likelihood of bleeding, presence of other high-risk factors such as diabetes, elderly/pediatric age group, and hemophilia. Thrombocytopenia in dengue is multifactorial. In the early stage, bone marrow hypocellularity followed later by immune-mediated destruction of platelets is proposed as the mechanisms for thrombocytopenia. ${ }^{[4]}$ Immune-mediated destruction of platelets in DF may lead to refractoriness, poor, or short-term response to platelet transfusions.

\section{Study aim}

The aim of this study was to study the effect of introduction of single-donor apheresis platelets (SDAPs) on dengue management. Patient profile and overall impact of this transfusion support on further requirement of platelet transfusions, length of stay, and cost of stay in hospital were studied.

\section{Materials and Methods}

This was a retrospective study conducted on clinically suspected cases of dengue infection attending the inpatient department of a teaching hospital of North India, between August 2013 and December 2015.

According to specific inclusion criteria, 622 clinically suspected patients with fever (presenting within 5-7 days of onset with body temperature above $100^{\circ} \mathrm{F}$ at the time of blood sample collection) and fulfilling the case definition criteria of DF/DHF/DSS of WHO and who also received platelet transfusions were included in this study. ${ }^{[6]}$

\section{Statistical analysis}

Clinical data, reports of hematological investigation, transfusion request forms, platelets requirements, and data obtained from daily follow-up and blood bank records were analyzed using IBM SPSS version 20 (IBM Corp, Armonk, NY).

\section{Results}

Six hundred and twenty-two dengue patients admitted between August 2013 and December 2015 received platelet transfusions, i.e., 364 patients received platelet transfusions in the year 2013 and 258 in the year 2015. All the dengue patients were divided into five age groups, i.e., $<15$ years, $16-30$ years, $31-45$ years, $46-60$ years, and $>61$ years. The largest number of patients was from the $31-45$ years age group. The youngest patient was 4 years old and the oldest was 86 years old [Table 1]. There was no significant difference between number of random donor platelets (RDPs) transfused with respect to these age groups $(P=0.5)$, but the mean number of RDP transfused was maximum in patients $<15$ years of age (5.9).

Out of the total 622 patients, 452 were male and 170 were female. Average number of RDP units transfused to male

Table 1: Age-wise distribution of dengue patients

\begin{tabular}{lcc}
\hline $\begin{array}{l}\text { Age group } \\
\text { (years) }\end{array}$ & $\begin{array}{c}\text { Number of } \\
\text { patients (\%) }\end{array}$ & $\begin{array}{c}\text { Average number } \\
\text { of RDP transfused }\end{array}$ \\
\hline$<15$ & $13(2.1)$ & 5.9 \\
$16-30$ & $183(29.4)$ & 5.1 \\
$31-45$ & $203(32.6)$ & 5.2 \\
$46-60$ & $165(26.5)$ & 4.7 \\
$>61$ & $58(9.3)$ & 4.7 \\
Total & $622(100)$ & 5 \\
\hline
\end{tabular}

$\mathrm{RDP}=$ Random donor platelet 
patients (mean value $=5.16$ ) was higher as compared to female patients (mean value $=4.56$ ) and as $P=0.03$, this difference was statistically significant.

A total of 188 (30.3\%) patients received platelet transfusions at platelet count $<20,000 /$ cumm and $385(61.9 \%)$ at $21,000-40,000 / \mathrm{cumm}$. Due to the presence of additional risk factors (such as diabetes, hypertension, elderly/pediatric age group, liver disease), 49 (7.9\%) patients received platelet transfusions at platelet count $>41,000 /$ cumm [Table 2]. None of the patients received platelet transfusion at platelet count $>100,000$ /cumm, and neither any of the admitted patients died during hospitalization (mortality being $0 \%$ ).

Patients were divided into four groups depending on their platelet count $(<10,000,11,000-20,000,21,000-40,000$, $>41,000$ /cumm each) at the initiation of transfusions and the average number of RDP transfused in each group was calculated. From this grouping, those patients receiving SDAP only were excluded from the study. There was a significant association between platelet count and the number of RDP received. On applying Chi-square test (Chi-square value was 111) $P<0.001$ which was highly significant indicating that lower the platelet count, more will be the requirement for platelet transfusions [Table 2]. Furthermore, there was a significant difference between these four groups with respect to the number of RDP used on applying one-way ANOVA test $(P<0.001)$ supporting the fact that as the platelet count dropped, the requirement for platelet transfusions increased proportionately.

SDAPs were introduced during second dengue outbreak, i.e., in 2015. Dengue patients admitted and receiving

Table 2: Lowest platelet count at which transfusion initiated and the number of random donor platelet transfused

\begin{tabular}{lcc}
\hline Platelet count & $\begin{array}{c}\text { Number of } \\
\text { patients (\%) }\end{array}$ & $\begin{array}{c}\text { Average number } \\
\text { of RDP transfused }\end{array}$ \\
\hline$<10,000$ & $29(4.7)$ & 7.3 \\
$11,000-20,000$ & $159(25.6)$ & 6.7 \\
$21,000-40,000$ & $385(61.9)$ & 4.4 \\
$>41,000$ & $49(7.9)$ & 3.3 \\
Total & $622(100)$ & 5.01 \\
\hline
\end{tabular}

$\mathrm{RDP}=$ Random donor platelet platelet transfusions in 2015 were divided into three major groups depending on the type of platelet transfusions received, i.e., those receiving RDP only (Group 1), those receiving RDP and SDAP both (Group 2), and those receiving SDAP only (Group 3). Platelet count at the time of initiation of platelet transfusions was calculated in these three transfusion groups and the difference was highly significant $(P<0.001)$. On applying post hoc test, least significant difference (LSD), there was significant difference in the platelet count at the time of initiation of platelet transfusions between Group 1 and $2(P<0.01)$ and Group 1 and $3(P=0.024)$ though there was no significant difference between Groups 2 and $3(P=0.47)$. Average platelet count at which platelet transfusion initiated was $25,703 /$ cumm. Average number of RDP units transfused in 2013 was 5.4 and 4.3 in 2015 and this difference was highly significant $(P<0.01)$ which was probably due to the reason that in 2015, SDAPs were also transfused to patients with lower platelet count and those with additional high-risk factors. The average rise in platelet count after SDAP transfusions was 46,040/cumm [Table 3].

Length of stay and total cost of stay in hospital were calculated among these three groups. Mean length of stay in hospital was similar for those receiving RDP only (Group 1) and those receiving RDP and SDAP both (Group 2), i.e., 5.48 versus 5.54, respectively, while that for SDAP only (Group 3) was quite lower 3.6 [Table 3]. When we compare these three transfusion groups together using ANOVA test, there was no significant difference between length of stay in hospital $(P=0.10)$, but on applying post hoc test (LSD), there was significant difference between length of stay between RDP (Group 1) and SDP group (Group 3) $(P=0.037)$. Average cost (Indian rupee) of treatment and stay among these three groups (1, 2, and 3) was Rs. 18,565 , Rs. 28,689 , and Rs. 24,512, respectively [Table 3]. On applying ANOVA test, the difference in the cost was highly significant $(P<0.001)$. Furthermore, on applying post hoc test (LSD), there was significant difference in the cost between Group 1 and 2 $(P<0.001)$ and Group 1 and $3(P=0.002)$ while there was only mild significant difference between Group 2 and $3(P=0.059)$. It can be concluded from these findings that transfusion of SDP increases the total cost for patient, but it also significantly reduces the length of stay in hospital.

Table 3: Platelet count at the initiation of platelet transfusion, length of stay, and total cost of stay in hospital in 2015

\begin{tabular}{lcccc}
\hline Transfusion group & Number of patients & $\begin{array}{c}\text { Platelet count at the } \\
\text { initiation of transfusions }\end{array}$ & $\begin{array}{c}\text { Mean length } \\
\text { of stay }\end{array}$ & $\begin{array}{c}\text { Mean cost of stay and } \\
\text { treatment (INR) (Rs) }\end{array}$ \\
\hline RDP (Group 1) & 185 & $27,502.7$ & 5.48 & $18,565.4$ \\
RDP + SDP (Group 2) & 48 & $20,562.5$ & 5.54 & $28,689.2$ \\
SDP (Group 3) & 25 & $22,400.0$ & 3.68 & $24,512.4$ \\
Total & 258 & $25,703.1$ & 5.32 & $21,025.2$ \\
\hline
\end{tabular}

$\mathrm{RDP}=$ Random donor platelet, SDP=Single-donor platelet, INR=Indian rupee 


\section{Discussion}

Most of dengue cases were clinically indistinguishable from other febrile illnesses and could be missed lacking the clinical suspicion and timely diagnosis. ${ }^{[7]}$ In most countries, dengue epidemics are reported to occur during the warm, humid, and rainy seasons, which favor abundant mosquito growth and shorten the extrinsic incubation period as well. ${ }^{[8,9]}$ The present study also focused on two consecutive dengue epidemics in the year 2013 and 2015, both of which started during the beginning of monsoon season of North India and ended with the beginning of winter season. Dengue is a viral hemorrhagic fever (VHF) and there is a need to develop standardized clinical protocols for the VHF clinical case management that integrate infection control measures with comprehensive care for patients. ${ }^{[10]}$

During explosive outbreaks of dengue, there is sudden surge in demands of platelet products. ${ }^{[11]}$ High-risk patients having platelet count $<20,000 /$ cumm with risk of bleeding require prophylactic platelet transfusion and those with platelet count between 21,000 and $40,000 /$ cumm are in moderate risk and require platelet transfusion only if they have any hemorrhagic manifestation and other superadded conditions. ${ }^{[12]}$ With a defensive approach in the present study, maximum numbers of transfusions were initiated at platelet counts of 21,000-40,000/cumm (61.9\%) which was a moderate risk category, and probably, this was the reason for zero mortality at our hospital.

Although DHF is primarily an illness of children, surveillance studies in recent years in several countries have reported increasing age among infected patients. ${ }^{[13,14]}$ The present study also supported the recent trend of increasing age as maximum number of patients was in the age group of $31-45$ years (32.6\%) and only $2.1 \%$ patients were younger than 15 years. However, the severity of illness was more among children $<15$ years as indicated by the mean number of RDP transfused was maximum in this age group (5.9), and thus, it was one of the high-risk groups for dengue patients.

Managing a severe dengue infection is a challenge, especially when complicated by other comorbidities. Host factors such as extremes of age, female sex, ethnicity, and comorbidities such as diabetes and hypertension are associated with severe disease. ${ }^{[15,16]}$ In the present study, maximum number of platelet transfusions received was associated with pediatric age group (5.9) and male sex (5.19). Platelet transfusions in few cases with associated comorbid illnesses $(7.9 \%)$ were initiated at higher platelet counts even at $>41,000 /$ cumm. This approach reduced the patient suffering and prevented mortality in the present study. As mentioned by the WHO Fact Sheet 2008, when adequately treated, the mortality rate for DHF can be as low as $1 \%$; however, if untreated, it can exceed $20 \%{ }^{[17]}$ Therefore, there is a need for the development of specific guidelines for transfusion of blood components, constant interaction and coordination among clinicians and transfusion specialists, and a regular medical audit to review the optimal utilization of blood components during such hemorrhagic outbreaks. ${ }^{[18]}$

Extensive consumption of blood components, especially platelet products, during DF outbreaks depletes the inventory of blood centers. Further, lack of knowledge, absence of evidence-based guidelines, and panic-like situation lead to flooding of transfusion services with blood and component requests. ${ }^{[19]}$ Many times, the prescription for blood components is not based on medical rationale, but as a response to an intense social pressure on the treating physicians by the patients and their relatives. ${ }^{[12]}$ This "syndrome" of chasing platelet count in dengue patients who were otherwise completely asymptomatic and improving was labeled as "Dengue panic syndrome" by Ahluwalia et al. The most untoward effect of this syndrome is sudden surge in platelet transfusion requisitions based only on platelet counts. ${ }^{[11,20]}$ In a randomized controlled trial by Khan et al., almost half the patients showed no response to a high-dose platelet transfusion. Platelet transfusion did not prevent development of severe bleeding or shorten time to cessation of bleeding and was associated with significant side effects. ${ }^{[21]}$ On the contrary, Jayashree et al. study concluded that a recovery from thrombocytopenia was associated with clinical improvement while further fall in platelet counts was associated with fatality. ${ }^{[22]}$ The present study also supported the findings of Jayashree et al. and Murali Mohan et al. that timely platelets transfusions in cases of DHF and DSS helped prevent severe hemorrhagic complications. ${ }^{[22,23]}$ It was quite evident that transfusion of high-dose platelets (SDAP) at the initiation of transfusion therapy compared to low-dose platelets (RDP) reduced the need to repeatedly transfuse RDP, fasten the recovery, and thus reduced the length of stay in hospital (5.48 vs. 3.68) but increased the overall cost of stay and treatment $(P=0.002)$. Group 2 patients who received RDP and SDAP both due to certain reasons such as nonavailability of donor suitable for apheresis donation or financial constraints at the beginning of the treatment in spite of lower platelet count (mean 20,562/cumm) and therefore, initially received RDP but later were transfused SDAP for continuously lowering platelet count or hemorrhagic complications. In these patients, the total length of stay (5.54) and cost of stay and total number of platelet transfusions received were more compared to Group 3 and 1 which supported the fact that high-dose 
platelet transfusions are much beneficial than repeated low-dose platelet transfusions in high-risk groups.

There are certain limitations of apheresis procedure (SDAP). It requires a donor who should meet the criteria required for the procedure and also a special kit that cannot be afforded by those with poor financial backgrounds. ${ }^{[24]}$ Due to the high cost and more technical expertise required, SDAP must be recommended to selected patients either when platelet-rich plasma and platelet concentrates (buffy coat-pooled platelets) in adequate doses are not available in the inventory. ${ }^{[25]}$ Due to the similar reasons in the present study, SDAPs were transfused to those patients who had a very low platelet count, had superimposed risk factors for bleeding, and had the affordability for this procedure. Transfusion of SDAP in these patients resulted with significant positive findings such as immediate and sustained rise in platelet count (average increase of 46,040 /cumm); shortening of total length of stay in hospital and early recovery; lower or no requirement of RDP; minimum exposure to multiple donors; and thus reduced risk of transfusion transmitted diseases and adverse transfusion reactions (such as allergic, transfusion-associated circulatory overload, transfusion-related acute lung injury). Moreover, the biggest advantage was that overall mortality among dengue patients was zero.

\section{Conclusion}

Inappropriate platelet transfusions during dengue outbreak is a widely reported concept in literature, but demarcation of appropriate transfusion is a very challenging job when dealing with such a fatal but curable disease if appropriately managed. Hence, providing high-dose platelets such as SDAPs to dengue patients at appropriate stage (constantly falling or very low platelet count, hemorrhagic complications, superadded risk factors) can reduce further requirement of platelet transfusions, fasten the recovery, reduce the hospital stay, lower the risk of transfusion-associated adverse reactions and thus, lowering the overall associated morbidity and mortality.

\section{Financial support and sponsorship Nil.}

\section{Conflicts of interest}

There are no conflicts of interest.

\section{References}

1. Gubler DJ, Clark GG. Dengue/dengue hemorrhagic fever: The emergence of a global health problem. Emerg Infect Dis 1995;1:55-7.
2. Teo D, Ng LC, Lam S. Is dengue a threat to the blood supply? Transfus Med 2009;19:66-77.

3. Tai DY, Chee YC, Chan KW. The natural history of dengue illness based on a study of hospitalised patients in Singapore. Singapore Med J 1999;40:238-42.

4. Chairulfatah A, Setiabudi D, Agoes R, Colebunder R. Thrombocytopenia and platelet transfusions in dengue hemorrhagic fever and dengue shock syndrome. WHO Dengue Bull 2003;27:141-3.

5. Chuansumrit A, Tangnararatchakit K. Pathophysiology and management of dengue hemorrhagic fever. Transfus Altern Transfus Med 2006;8:3-11.

6. World Health Organization. Guidelines for Treatment of Dengue Fever/Dengue Haemorrhagic Fever in Small Hospitals. Recognition of Dengue Fever/Dengue Haemorrhagic Fever (DF/ DHF). Grading the Severity of Dengue Infection. New Delhi: World Health Organization; 1999. p. 2-3.

7. Ahmed NH, Broor S. Dengue fever outbreak in Delhi, North India: A clinico-epidemiological study. Indian J Community Med 2015;40:135-8.

8. McBride WJ, Bielefeldt-Ohmann H. Dengue viral infections; pathogenesis and epidemiology. Microbes Infect 2000;2:1041-50.

9. Katyal R, Singh K, Kumar K. Seasonal variations in A. aegypti population in Delhi, India. Dengue Bull 1996;20:78-81.

10. Ippolito G, Feldmann H, Lanini S, Vairo F, Di Caro A, Capobianchi MR, et al. Viral hemorrhagic fevers: Advancing the level of treatment. BMC Med 2012;10:31.

11. Chaurasia R, Zaman S, Chatterjee K, Das B. Retrospective review of platelet transfusion practices during 2013 dengue epidemic of Delhi, India. Transfus Med Hemother 2015;42:227-31.

12. Makroo RN, Raina V, Kumar P, Kanth RK. Role of platelet transfusion in the management of dengue patients in a tertiary care hospital. Asian J Transfus Sci 2007;1:4-7.

13. Gupta E, Dar L, Kapoor G, Broor S. The changing epidemiology of dengue in Delhi, India. Virol J 2006;3:92.

14. Khan E, Siddiqui J, Shakoor S, Mehraj V, Jamil B, Hasan R. Dengue outbreak in Karachi, Pakistan, 2006: Experience at a tertiary care center. Trans R Soc Trop Med Hyg 2007;101:1114-9.

15. Pang J, Salim A, Lee VJ, Hibberd ML, Chia KS, Leo YS, et al. Diabetes with hypertension as risk factors for adult dengue hemorrhagic fever in a predominantly dengue serotype 2 epidemic: A case control study. PLoS Negl Trop Dis 2012;6:e1641.

16. Figueiredo MA, Rodrigues LC, Barreto ML, Lima JW, Costa MC, Morato $\mathrm{V}$, et al. Allergies and diabetes as risk factors for dengue hemorrhagic fever: Results of a case control study. PLoS Negl Trop Dis 2010;4:e699.

17. World Health Organization. Dengue and Dengue Haemorrhagic Fever. WHO Fact Sheet. Geneva: WHO; 2008.

18. Pallavi P, Ganesh CK, Jayashree K, Manjunath GV. Unfurling the rationale use of platelet transfusion in dengue Fever. Indian J Hematol Blood Transfus 2011;27:70-4.

19. Kaur P, Kaur G. Transfusion support in patients with dengue fever. Int J Appl Basic Med Res 2014;4 Suppl 1:S8-12.

20. Ahluwalia G, Sharma SK. Dengue: Current trends and challenges - An Indian perspective. J Assoc Physicians India 2004;52:561-3.

21. Khan Assir MZ, Kamran U, Ahmad HI, Bashir S, Mansoor H, Anees SB, et al. Effectiveness of platelet transfusion in dengue fever: A randomized controlled trial. Transfus Med Hemother 2013;40:362-8.

22. Jayashree K, Manasa GC, Pallavi P, Manjunath GV. Evaluation of platelets as predictive parameters in dengue fever. Indian J Hematol Blood Transfus 2011;27:127-30.

23. Murali Mohan KK, Rao BB, Raghuram C, Gunnam S, Dharmateja D, 
Gopidi A. A clinical study of dengue fever cases: NRI Medical College, Chinakakani, Andhra Pradesh, South India. Int J Med Public Health 2015;5:367-71.

24. Chatterjee K, Coshic P, Borgohain M, Agarwal N. Experience of buffy coat pooling of platelets as a supportive care in thrombocytopenic dengue patients: A prospective study. Asian
J Transfus Sci 2014;8:89-91.

25. Singh RP, Marwaha N, Malhotra P, Dash S. Quality assessment of platelet concentrates prepared by platelet rich plasma-platelet concentrate, buffy coat poor-platelet concentrate (BC-PC) and apheresis-PC methods. Asian J Transfus Sci 2009;3:86-94. 